\title{
Formation à la communication scientifique et interpersonnelle en anglais pour la santé : quelle prise en charge en formation initiale et en formation continue? Vers une harmonisation de l'enseignement en France métropolitaine
}

Teaching of scientific and interpersonal communication in English for medical purposes in French medical schools: what should be taught during the medical studies and in continuing medical education sessions? Toward a national syllabus

\section{Rita CAMARÉ-Querci ${ }^{1}$, Félicie PASTore ${ }^{2}$, Jonathan Broner ${ }^{3}$ et Maelle AllibE ${ }^{4}$}

1 Vice-décanat et commission d'anglais, Faculté de médecine Toulouse-Purpan, Toulouse, France

2 Commission d'anglais, Faculté de médecine Toulouse-Purpan, Toulouse, France

3 Association corporative des étudiants en médecine de Toulouse, Faculté de médecine Toulouse-Purpan, Toulouse, France

4 Association nationale des étudiants en médecine de France (ANEMF)

Manuscrit reçu le 3 juin 2008 ; commentaires éditoriaux formulés aux auteurs le 10 février 2010 ; accepté pour publication le 16 février 2010

Mołs clés :

Langue étrangère ; psychologie cognitive ; pédagogie médicale
Résumé - Contexte : L'enseignement de l'anglais dans les facultés de médecine est obligatoire depuis 1992 mais cette disposition est diversement respectée. Buts : Bilan et perspectives ouvertes par le partenariat entre l'Association nationale des étudiants en médecine de France (ANEMF) et le Groupe d'étude et de recherche en anglais de Spécialité (GERAS) membre de la Société des anglicistes de l'enseignement supérieur (SAES). Matériel : Exploitation de différents travaux publiés (articles, DEA, manuels), enquêtes, réunions GERAS-ANEMF. Résultats : Les enseignants de médecine, d'abord confrontés à la difficulté d'introduire un nouvel enseignement, avec une diminution du volume horaire des matières médicales et la nécessité de déployer des ressources matérielles et humaines, participent à présent à l'enseignement de l'anglais. Les difficultés initiales rencontrées par les enseignants d'anglais sont compensées par des succès visibles dans certaines facultés. L'enseignement de l'anglais, loin d'être au mieux une remédiation, au pire une perte de temps, vise une compétence effective en communication écrite, orale et interpersonnelle des professionnels de la santé du XXI ${ }^{\mathrm{e}}$ siècle. Les étudiants, longtemps hostiles à la réforme, montrent désormais leur intérêt et souhaitent une harmonisation nationale des contenus, conscients de la nécessité pour eux de pouvoir 
Keywords:

Foreign language; cognitive psychology; medical pedagogy travailler en anglais. Conclusions : La mise en œuvre et le succès de la Charte d'enseignement de l'anglais élaborée par l'ANEMF et le GERAS dépendront étroitement du soutien apporté par les autorités compétentes de chaque faculté à ce projet, dont la visée est une aide au déroulement de la carrière et de l'activité clinique aussi bien qu'au développement personnel des futurs professionnels de la santé.

Abstract - Context: English has been a compulsory subject since 1992, yet this has been unequally implemented. Aim: To take stock of the work done during the past 15 years and assess the new perspectives offered by a recent partnership between the Association Nationale des Étudiants en Médecine de France (ANEMF, National Association of French Medical Students) and the Groupe d'Étude et de Recherche en Anglais de Spécialité (GERAS, a group of teachers of English for Medical Purposes, a Special Interest Group of the Société des Anglicistes de l'Enseignement Supérieur (SAES), Society of Teachers of English in Upper Education). Material: Articles, dissertations, workbooks, surveys, symposia reflecting the work of teachers in the Special Interest Group. Results: Professors of medicine, first confronted with the difficult task of introducing a new subject, implying a reduction of the total number of teaching hours of medicine and the necessity of finding human and material resources, are now often actively involved in the English syllabus. The initial difficulties met by the teachers of English for medical purposes are now compensated for by the visible achievements in some medical schools. The teaching of English, far from being mere remediation at best or a loss of time at worst, aims to provide the medical professionals of the 21 st century with effective writing and speaking skills. The students, long reluctant to the reform, are now aware of the necessity for them to be able to work in English. Starting to show their interest, they ask for a national syllabus, described in a national chart. Conclusions: The success of this project, whose aim is to provide a help in the career, clinical activity as well as personal development of future health professionals, prepared and agreed by ANEMF and GERAS representative members, now depends on the backup provided by each medical school's board.

\section{Introduction}

Chacun s'entend pour dire qu'il est indispensable aux professionnels de la santé de pouvoir travailler en anglais, que ce soit pour interroger un patient au cours d'une consultation, effectuer la remise à jour constante des connaissances par la lecture des périodiques spécialisés, publier un article dans une revue internationale, assister à un congrès international, poser des questions après une communication orale ou présenter soi-même les résultats de sa recherche puis faire face à la séance de questions-réponses, redoutable pour des francophones. Ces compétences requièrent un entraînement spécifique, qui n'est bien sûr pas dispensé dans les établissements d'enseignement du second degré et qui doit être assuré au sein de la faculté de médecine. Quelle place faut-il alors donner à l'enseignement de l'anglais en faculté de médecine, que faut-il enseigner et comment?

\section{Problématique}

Historique

L'enseignement de l'anglais est passé du statut d'enseignement facultatif à celui d'enseignement obligatoire, avec des modalités d'application extrêmement variables selon les facultés, après l'arrêté du 18 mars 1992 dont l'article 18 postulait : «L'enseignement des langues étrangères doit représenter l'équivalent d'au moins 120 heures, soit sous forme d'un enseignement spécifique, soit intégré à celui d'autres disciplines. S'il n'a pas été dispensé en première année du premier cycle, il doit obligatoirement être organisé en seconde année du premier cycle et en première année du deuxième cycle $[. .$.$] ».$ 


\section{Évolution de la situation de 1992 à 2006}

La prise en compte de ce besoin de formation en anglais pour la médecine au cours des quinze dernières années peut-être examinée au regard du double point de vue des enseignants et de celui des étudiants.

\section{Ce qu'en disent les enseignants}

Les enseignants concernés sont au premier chef ceux du groupe d'étude et de recherche en anglais de spécialité (GERAS), composante de la Société des anglicistes de l'enseignement supérieur (SAES). Les travaux universitaires effectués dans le cadre de l'équipe «Anglais de spécialité, politique et didactique de l'anglais »(ASPDA, ex EA 2025), à l'Université de Bordeaux $2^{[1,2]}$, les communications présentées lors de congrès ${ }^{[3,4]}$, ainsi que les différents articles publiés dans la presse médicale ${ }^{[5]}$ permettent de suivre l'évolution de la situation et du statut de l'enseignement de l'anglais en faculté de médecine.

Des enquêtes quantitatives ont été réalisées au cours des quinze années considérées depuis l'introduction de la réforme, à intervalle à peu près régulier de trois ans, dans le cadre de ces travaux universitaires. Leurs résultats et conclusions sont corroborés par ceux de l'enquête menée dans le cadre du projet d'harmonisation de l'enseignement de l'anglais présenté au bureau de la Conférence des doyens des facultés de médecine par des représentants du GERAS le 19 octobre $2004^{[6]}$.

Une nouvelle fois, ce qui ressortait de cette enquête était la grande disparité des volumes horaires, des moyens mis en œuvre et des situations d'enseignement. Outre cette disparité, on remarquait que, dans certaines facultés de médecine, des cours sont dispensés en amphithéâtre, ce qui limite l'interactivité possible. De plus, des différences existaient relativement aux plages horaires (car nombre de facultés placent l'anglais tard dans la journée), aux moyens mis en œuvre (de nombreuses facultés n'ont ni équipement, ni supports et beaucoup manquent d'intervenants), au statut des enseignants et à leur degré de formation (74\% d'entre eux s'estiment peu ou mal formés pour faire face à leur mission). On observait en conséquence un manque de motivation des étudiants dans de nombreuses facultés.

Le rapport concluait que dans la majorité des facultés de médecine les textes officiels n'étaient pas réellement appliqués, qu'il existait une grande disparité de traitement des étudiants vis-à-vis de l'enseignement de l'anglais et que, dans l'intérêt de la formation des futurs médecins, une harmonisation paraissait souhaitable au niveau national, qui tiendrait compte de l'hétérogénéité des situations. Le rapport proposait qu'une réflexion commune soit organisée entre la conférence des doyens et les représentants des enseignants d'anglais, sur la base de programmes cohérents déjà mis en place dans certaines universités. Mais le dossier était resté en l'état.

Pourtant, les efforts d'harmonisation ont été constants tout au long de ces quinze années, bloqués en formation initiale par les différences d'exercice déjà mentionnées, mais possibles au niveau de la formation continue, où une harmonisation des contenus a été effectuée dans un certain nombre d'universités qui proposent le diplôme inter-universitaire d'anglais pour la médecine (DIVAM), ouvert en 1998, et sa version en ligne, le Diplôme inter-universitaire d'anglais pour les professions de santé (DIVAS), ouverte en 2002 pour rendre possible la formation en langue anglaise des professionnels de santé sur tous les points du territoire ${ }^{[7,8]}$.

\section{Ce qu'en disent les étudiants}

Les étudiants ont été sollicités par l'intermédiaire de l'Association nationale des étudiants en médecine de France (ANEMF). En février 1997, dans le «Rapport sur la formation médicale initiale tant théorique que pratique », ils formulaient un jugement sévère : «L'enseignement de l'anglais, très important puisque c'est la langue de référence dans notre discipline, ne nous apparaît pas adapté actuellement. En effet, celui-ci est souvent réalisé par des professeurs agrégés (PRAG) pour lesquels la médecine est totalement étrangère. Les cours sont donc également peu suivis et peu instructifs. ». On lisait dans les 
«Propositions de refonte de la formation médicale initiale » : «L'anglais étant la langue de référence en médecine, il apparaît comme primordial qu'une approche de l'anglais médical soit dispensée. Celuici doit se faire tout au long des études, par exemple par quelques cours en anglais ou par l'analyse d'articles en anglais. Ces cours doivent être donnés par des enseignants de médecine pratiquant couramment l'anglais ».

En juin 2006, soit près de 10 ans après ce rapport, un document intitulé «Améliorer l'enseignement de l'anglais au cours du cursus médical » a été présenté au cours des «Journées d'été de l'ANEMF ». Ce document faisait suite à une enquête menée par l'ANEMF (qui ne précise cependant pas quelle méthode a été adoptée ${ }^{[9]}$. Les conclusions en sont que la part de l'anglais est souvent négligée et que peu de facultés proposent effectivement 120 heures. Le rapport stipule que «seules trois facultés sont en mesure d'assurer cet enseignement, Toulouse-Purpan, Nice et Dijon. Les raisons qui expliquent ce manque sont certainement nombreuses : manque de temps dans les emplois du temps déjà chargés, manque de salles, manque d'accès à des équipements spécialisés du type laboratoire de langues, manque d'enseignants. Par ailleurs le niveau est souvent faible, comme l'intérêt de l'enseignement. La plupart du temps, les enseignants qui assurent les cours d'anglais sont issus des facultés de langue et n'ont que peu de rapport avec le monde médical. Aussi, les thèmes abordés intéressent rarement les étudiants qui pensent perdre leur temps en assistant aux enseignements, qui sont trop scolaires, avec pour conséquence une démotivation des étudiants ».

L'ANEMF propose d'adopter un enseignement en petits groupes (15 personnes), d'assurer une remise à niveau pour les plus faibles, préconise la «médicalisation» de l'enseignement, avec des cours médicaux en anglais, demande que l'enseignement de l'anglais utilise au maximum les nouvelles technologies de l'information et de la communication (NTICE), enfin qu'une validation cohérente et un contrôle des connaissances varié soit adoptés.

\section{Une convergence de points de vue}

Bien que l'analyse soit différente, les conclusions tirées des perspectives respectives des enseignants et des étudiants sont donc similaires. Il paraît indispensable de repenser l'enseignement de l'anglais dans la plupart des facultés de médecine et d'harmoniser les objectifs et les programmes au niveau national. Les étudiants sont à présent très conscients des besoins en communication médicale en anglais des professionnels de la santé et demandent qu'une formation adaptée à ces besoins soit également assurée sur tout le territoire métropolitain.

\section{Cadre conceptuel et principes pédagogiques directeurs}

La perspective socio-constructiviste

Le cadre théorique qui sous-tend les propositions qui suivent pour l'enseignement de l'anglais dans les facultés de médecine métropolitaine est le modèle socio-constructiviste, qui inscrit la formation dans le contexte professionnel de l'apprenant, ainsi que les travaux en psychologie cognitive et en psychologie humaniste, qui donnent une grande importance au monde intérieur de l'apprenant, à ses pensées, ses sentiments et ses émotions. Ces propositions intègrent aussi les travaux des experts en pédagogie médicale, en particulier ceux des chercheurs nordaméricains et des membres de la commission de pédagogie médicale de la Conférence internationale des doyens et des facultés de médecine d'expression française $(\mathrm{CIDMEF})^{[10-13]}$, notamment les travaux sur les conditions du transfert des connaissances, déterminé par la façon dont les étudiants acquièrent, organisent et mémorisent celles-ci pour pouvoir ensuite les mobiliser judicieusement. Il a été montré que les connaissances d'action ne peuvent être encodées que dans l'action car la similarité entre les conditions de l'encodage et celles de l'utilisation facilite le rappel. Il en résulte que l'apprentissage contextualisé est une condition fondamentale pour l'encodage efficace. Il a été montré d'autre part que l'effet de la pratique sur la mémoire est extrêmement 
spécifique. Il y a une corrélation entre le nombre de fois où une connaissance a été rappelée à partir de la mémoire à long terme vers la mémoire de travail, d'une part, et la facilité avec laquelle on peut la rappeler à nouveau, d'autre part.

\section{Les principes directeurs pour l'enseignement}

Il s'agit donc de mettre en œuvre des activités d'enseignement et d'apprentissage de l'anglais à visée professionnelle, qui permettent la mise en œuvre du processus individuel, actif et constructif, qui se produit lorsque l'étudiant traite activement l'information nouvelle, modifiant ainsi sa structure cognitive, qui est au cœur de l'apprentissage. Le changement pédagogique préconisé pour l'enseignement de l'anglais en faculté de médecine est semblable à celui qui s'est déjà opéré dans l'enseignement de la médecine dans un certain nombre de facultés. Il vise à :

- placer l'apprenant au centre de son apprentissage ;

- développer son autonomie et sa responsabilité vis-à-vis de son apprentissage ;

- développer sa motivation et sa confiance dans ses capacités d'apprenant efficace ;

- développer sa conscience de la nécessité de prendre en charge les moyens de maintenir et accroître sa compétence ;

- développer son ouverture, sa disponibilité à saisir les occasions d'apprendre.

Les principes directeurs pour l'évaluation des apprentissages

Les différents domaines à évaluer sont respectivement le domaine cognitif, qui fait référence à la dimension du savoir (linguistique, vocabulaire et grammaire), le domaine psychomoteur, qui concerne le savoir-faire et la dimension pragmatique (capacités de communication) et le domaine affectif, relatif au savoir-être et à la dimension sociopsychologique (prise de parole en public et communication interpersonnelle).
Le savoir linguistique indispensable dans le domaine de la santé peut être évalué à partir d'un programme de vocabulaire et de grammaire pour chacune des années d'étude, selon le principe d'un apprentissage «en spirale» basé sur le manuel «L'anglais pour la santé : exercices d'appropriation de la langue à visée professionnelle » où chaque domaine est revisité chaque année en complexifiant les exercices proposés ${ }^{[14]}$.

Les savoir-faire utiles aux futurs professionnels (rédiger et présenter une lettre, commenter des données chiffrées, prendre position et argumenter son point de vue, lire et comprendre un article de recherche aussi vite en anglais qu'en français, rédiger un article de vulgarisation scientifique à partir d'un article de recherche, rédiger un abstract, faire une communication orale avec le support d'un diaporama) doivent être évalués de façon progressive tout au long des années pendant lesquelles l'anglais est enseigné.

Le savoir-être lors d'une communication orale et de la séance de questions-réponses doit être évalué chaque année, tandis que les différences interculturelles à connaître et mettre en œuvre lors d'une consultation avec un patient anglophone sont abordées à partir des années d'études cliniques (deuxième - DCEM2 - et troisième - DCEM3 - années du deuxième cycle des études médicales).

\section{Le dispositif de formation mis en place à la faculté de médecine de Toulouse- Purpan}

La réforme adoptée en septembre 2006 à la faculté de médecine Toulouse-Purpan est basée sur les principes énoncés précédemment. Le programme, développé sur quatre ans, prend en compte les besoins et les demandes des apprenants et est doté des ressources techniques et humaines nécessaires. Cette réforme a été élaborée et adoptée par les représentants des étudiants et des enseignants d'anglais, la responsable de la commission d'anglais et le doyen de la faculté (ou unité de formation d'enseignement et recherche - UFR). 
Analyse de la situation d'enseignement et des besoins

Il a été nécessaire de prendre en compte les différents paramètres de la situation d'enseignement et d'effectuer une analyse des besoins de la population cible, des attentes des étudiants, des demandes des autorités compétentes de l'UFR médecine ainsi qu'une analyse des ressources matérielles et humaines mobilisables à court, moyen et long terme. Une des conditions indispensables au succès d'une telle entreprise est d'établir un partenariat étroit dans l'analyse et la prise de décisions entre les représentants des étudiants, les représentants des enseignants d'anglais et les autorités compétentes de la faculté de médecine.

\section{La réforme}

À la suite de cette analyse, la faculté de médecine de Toulouse-Purpan a décidé la mise en œuvre à la rentrée 2006-2007 d'un nouveau dispositif d'enseignement et d'un apprentissage de l'anglais à visée professionnelle. Le programme de $180 \mathrm{~h}$ en quatre ans $(60 \mathrm{~h}$ en deuxième année du premier cycle des études médicales - PCEM2 -, $60 \mathrm{~h}$ en première année du deuxième cycle des études médicales - DCEM1 -, 30 h en DCEM2 et 30 h en DCEM3) permet de passer progressivement de «l'anglais pour la médecine » en PCEM2 et DCEM1 à «la médecine en anglais », en DCEM2 et DCEM3.

Le manuel adopté, «L'anglais pour la santé : exercices d'appropriation de la langue à visée professionnelle » ${ }^{[14]}$, est la base commune à tous les groupes de travaux dirigés pour le programme de vocabulaire et de structures prioritaires en anglais pour les professions de santé, développé sur les quatre années. Tous les documents proposés (écrits, oraux et informatiques) sont spécifiques à la formation médicale et les sujets d'étude sont, dans toute la mesure du possible, choisis en rapport avec le programme médical de chacune des années. Au terme de la formation sur quatre ans, l'objectif est que les étudiants soient capables de travailler en anglais. L'enseignement utilise au maximum NTICE, en particulier

\begin{tabular}{|l|}
\hline \multicolumn{1}{c|}{ Encadré 1} \\
Objectifs de l'enseignement de l'anglais à la faculté \\
de médecine Toulouse-Purpan.
\end{tabular}

grâce au laboratoire de langues interactif qui a remplacé un matériel devenu obsolète.

Les enseignants de médecine répondent très favorablement à la demande qui leur a été faite de participer à l'enseignement de l'anglais, de PCEM2 à DCEM3. Ils interviennent dans chacune des années d'étude où ils font des conférences en anglais, et la session intensive de $20 \mathrm{~h}$ en une semaine en DCEM3 consiste en des séances d'apprentissage du raisonnement clinique en anglais animées par les médecins sur le modèle des cas cliniques proposés en français à l'examen classant national. Il s'agit véritablement d'un enseignement intégré médecine-anglais.

Pour chaque année, l'évaluation porte sur les connaissances théoriques et les connaissances procédurales. Enfin, chaque période d'enseignement fait l'objet d'une évaluation par les étudiants.

\section{Les modalités opérationnelles}

Des objectifs ont été fixés pour l'ensemble de la formation et pour chaque année d'études (encadré 1). 


\section{Encadré 2}

Répartition par année d'études des contenus d'enseignement de l'anglais à la faculté de médecine Toulouse-Purpan.

Deuxième année du premier cycle des études médicales (PCEM 2) : l'anglais pour la médecine

- Structures prioritaires et vocabulaire médical

- Méthodologie lecture rapide, commentaire de données chiffrées, lettres, communication orale support diaporama

Première année du deuxième cycle de études médicales (DCEM1) : l'anglais pour la médecine

- Structures prioritaires et vocabulaire médical

- Approfondissement des connaissances et savoir-faire acquis en PCEM2 + Initiation au Test English as a Foreign Language (TOEFL) pour séjour à l'étranger

Deuxième année du deuxième cycle de études médicales (DCEM2) : la médecine en anglais avec les enseignants de médecine et les médecins anglophones

- Structures prioritaires et vocabulaire médical

- Abstracts, communications orales, consultations, cas cliniques

Troisième année du deuxième cycle de études médicales (DCEM3) : la médecine en anglais avec les enseignants de médecine et les médecins anglophones

- Structures prioritaires et vocabulaire médical

- Rédaction de vulgarisation scientifique, apprentissage du raisonnement clinique (ARC), apprentissage par problèmes (APP), réunions de service, cas cliniques type «épreuves classantes nationales (ECN) »

Une progression logique a été adoptée, s'appuyant sur les acquis et complexifiant les tâches chaque année (encadré 2). Une évaluation des connaissances, des savoir-faire et des savoir-être a été instaurée à chaque étape de la formation (encadré 3). L'enseignement transversal médecine-anglais a été mis en œuvre.

Pour obtenir une large participation des enseignants de médecine à l'enseignement de l'anglais, condition indispensable pour avoir une réelle crédibilité et pour que la charge ne soit pas écrasante pour les enseignants qui acceptent d'y participer, l'enseignante de médecine responsable de la commission d'anglais a contacté tous ceux parmi ses collègues qui étaient susceptibles de participer à l'enseignement de l'anglais, même s'ils n'étaient pas bilingues et même s'ils étaient loin de l'être. En effet, l'implication de ces enseignants libère beaucoup les étudiants les moins avancés en anglais et relance leur

\begin{tabular}{|c|}
\hline $\begin{array}{c}\text { Encadré } 3 \\
\text { Modalités d'évaluation des apprentissages } \\
\text { de l'anglais à la faculté de médecine } \\
\text { Toulouse-Purpan. }\end{array}$ \\
$\begin{array}{c}\text { Savoirs : vocabulaire et structures prioritaires } \\
\text { Savoir-faire : } \\
\text { - Méthodologie de lecture rapide (« skimming » et } \\
\text { « scanning ») } \\
\text { - Commentaire de données chiffrées } \\
\text { - Lettres (mise en page, contenu, formules) } \\
\text { - Prise de position argumentée (essay) } \\
\text { - Communication orale avec diaporama } \\
\text { Savoir-être : communication non-verbale et com- } \\
\text { munication interpersonnelle lors de la consultation } \\
\text { « bedside manner })\end{array}$ \\
\hline
\end{tabular}




\section{Encadré 4}

Modalités d'intervention lors des conférences en amphithéâtre pour l'enseignement de l'anglais à la faculté de médecine de Toulouse-Purpan.

Contexte : la conférence remplace une séance de travaux dirigés ; donc la présence est obligatoire et contrôlée

Objectifs : compréhension de l'oral, prise de notes lors d'un congrès, prise de parole en public

Format : interventions courtes (10 à 15 minutes, donc deux ou trois parties) car la capacité des étudiants à se concentrer sur une communication en anglais l'impose.

Interactivité indispensable. Pour s'assurer de la bonne compréhension par chacun, il faut prévoir entre deux parties des activités de feed-back (quiz, questions appelant un court développement, sujet de réflexion, permettant une mise en commun par petits groupes, en anglais bien sûr - prévoir 10 minutes). Les enseignants, médecin et linguiste, circulent entre les groupes dans l'amphi en faisant fonction de «personnes ressources ».

Prise de parole en public : de façon à habituer dès le début les étudiants à ne pas souffrir du mal français (inhibition en public, à plus forte raison en anglais) : présentation du travail effectué par le petit groupe devant l'ensemble des étudiants - 10 minutes.

Commentaire de données chiffrées (pie-chart, bar-chart, graph ou table)

Diaporama imprimé (6 vignettes par page), qui sera distribué en fin de séance.

Questions à choix multiple (QCM), questions à réponse ouverte et courte (QROC) à compléter par l'étudiant pendant la séance, pour qu'il garde une trace du travail, ce qui lui permettra d'organiser ses révisions en vue de l'examen semestriel.

motivation. Un large réseau d'enseignants de médecine qui participent à l'enseignement de l'anglais s'est constitué, après définition des modalités de leur intervention (encadré 4). La prise en charge de la formation continue en anglais des professionnels de santé est assurée par le nouveau programme du (DIVAS) ${ }^{[15]}$ et les internes, chefs de clinique, professeurs et praticiens ainsi formés chaque année, rejoignent ensuite le réseau évoqué ci-dessus.

L'utilisation des NTICE a été renforcée, grâce à une amélioration de l'installation existante. Le nouveau laboratoire de langues est un élément clé de l'enseignement-apprentissage, qui guide progressivement les étudiants vers de plus en plus d'autonomie. L'apprentissage de l'anglais ne peut se limiter à la période des études de médecine et doit se poursuivre tout au long de la vie. L'autonomie acquise sera indispensable aux étudiants pour poursuivre seuls leur formation en anglais à l'issue de leurs études de médecine afin de ne pas perdre le bénéfice de l'effort consenti pendant les études. La participation active des enseignants du GERAS au campus numérique de l'Université médicale virtuelle francophone (UMVF) permet à tous de disposer de documents pertinents, spécialisés et actualisés. La grande variété et la richesse des ressources offertes sur internet par les périodiques médicaux aussi bien que la presse généraliste (articles, commentaire audio du sommaire de chaque numéro dans le New England Journal of Medicine ou le Journal of the American Medical Association, par exemple, émissions radiophoniques médicales, vidéos médicales thématiques et approfondissement possible des connaissance en direct, en anglais, à partir des sites des universités anglophones), permettent aux étudiants de prendre l'habitude d'utiliser les ressources de sites qu'ils pourront ainsi continuer à utiliser seuls.

Pendant les séances, la technique du déficit d'information (information gap), qui met les apprenants en situation authentique de communication, est largement utilisée dans le laboratoire de langues. Celui-ci permet en outre un travail en binôme par «téléphone » aussi bien qu'un travail comparatif classique pour améliorer le rythme, la 


\section{Encadré 5}

\section{Diplôme universitaire de communication en anglais dans les sciences de la santé (programme 2008-2009).}

DURÉE : 1 an

ORGANISATEUR : Dr Rita Camare-Querci, UFR Médecine Purpan

RESPONSABLE PÉDAGOGIQUE : Félicie Pastore, professeur agrégée UFR Langues

ACCÈS :

- Titulaires d'un diplôme de Docteur en Médecine, Odontologie, Pharmacie et Médecine vétérinaire

- Étudiants du $3^{\mathrm{e}}$ cycle des études médicales, odontologiques, pharmaceutiques et DFEG vétérinaires

- Titulaires d'un diplôme de Sage-femme

OBJECTIFS PÉDAGOGIQUES : acquérir 10 savoir-faire permettant de travailler en anglais. Au terme de la formation, les apprenants doivent pouvoir

- faire une consultation (interroger le patient, remplir la fiche observation)

- faire une réunion de service

- communiquer avec un confrère (lettre, téléphone : sigles, examens complémentaires)

- faire une communication orale (présentation de données chiffrées, support Powerpoint, commentaire de graphiques, communication verbale et non verbale)

- faire face à la séance de questions/réponses après la communication... et aux pauses café, repas

- participer à une table ronde (argumentation, accord/désaccord sans froisser susceptibilités)

- suivre une conférence en prenant des notes

- lire un article de recherche en anglais aussi vite qu'en français

- rédiger un abstract, rédiger une communication affichée

- rédiger un article de recherche (initiation à la publication)

Tous les documents (écrits, oraux et informatiques) proposés sont spécifiques à la formation médicale.

\section{PROGRAMME}

- Enseignement présentiel :

> 24 heures sur 3 jours de remise à niveau/approfondissement : anglais pour la médecine vocabulaire, phonologie et structures prioritaires en anglais pour les professions de santé, compréhension de l'oral, expression

> 64 heures sous forme de 3 séminaires intensifs de 3 jours consécutifs : médecine en anglais communication scientifique écrite et orale

- Entre les périodes d'enseignement présentiel : travail personnel guidé : vocabulaire, grammaire, rédaction scientifique, compréhension de l'oral, suivi téléphonique

\section{CONTRÔLE DES CONNAISSANCES - ÉVALUATION}

- Nul n'est admis à se présenter à l'examen final s'il n'a suivi avec assiduité l'enseignement en présentiel (minimum de $75 \%$ du temps d'enseignement)

- L'obtention du diplôme est validée par une note moyenne de 10/20 obtenue sur l'ensemble des épreuves écrites et orales

NOMBRE DE PARTICIPANTS : 12 minimum, 15 maximum par année universitaire

DATES ET LIEU DE LA FORMATION : Faculté de Médecine 37 allées Jules Guesde, Salle multimédia

- Lundi 12 au mercredi 14 janvier inclus de $9 \mathrm{~h}$ à $13 \mathrm{~h}$ et de $14 \mathrm{~h}$ à $18 \mathrm{~h}$

- Lundi 16 et mardi 17 février de 9 h à 13 h et de $14 \mathrm{~h}$ à 18 h, et mercredi 18 février de $9 \mathrm{~h}$ à $13 \mathrm{~h}$

- Lundi 23 et mardi 24 mars de 9 h à 13 h et de 14 h à 18 h, et mercredi 25 mars de 9 h à $13 \mathrm{~h}$

- Lundi 27 au mercredi 29 avril inclus de 9 h à 13 h et de 14 h à 18 h

\section{FRAIS D'INSCRIPTION :}

- Étudiants du $3^{\mathrm{e}}$ cycle : $300 €$

- Inscription individuelle : $700 €$

- Prise en charge par un organisme de formation continue : $900 €$

\section{INSCRIPTION :}

- Après entretien téléphonique en anglais

- Contacter avant le 15 octobre 2008 pastore@cict . fr 
prononciation des termes employés lors d'une communication orale et l'intonation (souvent difficile pour des français, qui ont tendance à utiliser l'intonation montante, donnant ainsi l'impression qu'ils posent sans cesse des questions, ce qui est fort gênant lorsqu'on veut affirmer la solidité de ses résultats).

\section{Évaluation préliminaire du dispositif de formation}

\section{L'évaluation des enseignements par les étudiants}

Cette démarche est systématique à la fin de chaque période d'enseignement. Les enquêtes de satisfaction ne sont pas encore courantes dans l'enseignement en France, de sorte que les réponses ne sont pas toujours informatives ni vraiment fiables. Néanmoins, leurs résultats indiquent un état d'esprit qu'il ne faut ni ignorer, ni négliger et l'introduction systématique de telles enquêtes après toute période d'enseignement est un gage de la communication nécessaire entre enseignants et enseignés.

Il s'agit de proposer un questionnaire rapide, ouvert et dont l'exploitation donne lieu à des modifications ultérieures éventuelles de l'enseignement pour répondre au mieux aux besoins des apprenants. Les rubriques du questionnaires ont été suggérées par les représentants toulousains de l'International Federation of Medical Students' Association (IFMSA) (encadré 6).

Impact du dispositif sur la formation des étudiants toulousains

Ce nouveau dispositif de formation a remporté tout d'abord l'adhésion puis la satisfaction des étudiants en formation initiale et en formation continue (DIVAS), comme en témoigne l'analyse des questionnaires remplis systématiquement par les apprenants à la fin de chaque période d'enseignement.

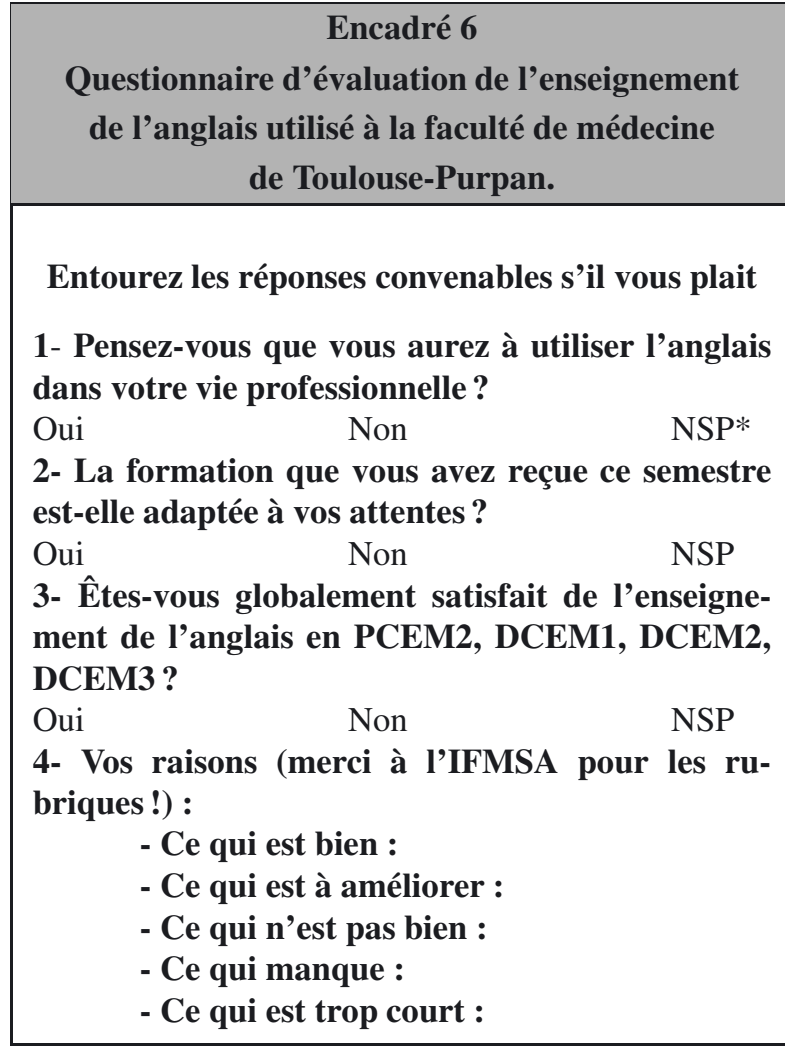

Impact du dispositif au niveau national

Les représentants toulousains de l'ANEMF ont eu l'occasion de faire part de leur expérience à leurs homologues lors de réunions nationales, qui ont alors suggéré un rapprochement des enseignants et des étudiants dans le cadre d'une réunion du GERAS. Les représentants nationaux de l'ANEMF et les enseignants d'anglais du GERAS, dont plusieurs ont déjà mis en œuvre dans leur UFR un enseignement de l'anglais qui s'appuie sur les mêmes bases théoriques $^{[16]}$, se sont ainsi mis à travailler ensemble à l'élaboration d'une proposition de charte commune d'enseignement de l'anglais.

La charte pour l'enseignement de l'anglais dans les facultés de médecine

La charte pour l'enseignement de l'anglais dans les facultés de médecine en France métropolitaine a été 
élaborée en partenariat entre le GERAS et les représentants nationaux de l'ANEMF, entre octobre 2006 et octobre 2007. Les travaux communs ont permis de réaffirmer l'importance de la maîtrise de l'anglais dans le contexte médical à l'écrit (lecture de revues spécialisées, d'articles de recherche), mais surtout à l'oral (interrogatoires de patients étrangers, cas cliniques concrets au service d'urgence, communications scientifiques). La version définitive de la charte a été agréée en réunion du 8 octobre $2007^{[17]}$.

Perspectives

Pour que cette harmonisation d'un enseignement d'anglais efficace devienne effective sur tout le territoire métropolitain, les étudiants et les enseignants ont à présent besoin que les instances dirigeantes des facultés de médecine deviennent véritablement partie prenante du projet. Les étapes à franchir sont : la validation des programmes et des modalités de contrôle des connaissances et savoir-faire en partenariat entre les étudiants, les enseignants d'anglais et le conseil d'UFR; la satisfaction des besoins en ressources matérielles et humaines; la constitution d'un réseau pédagogique qui permette un enseignement transversal.

\section{Conclusions}

Dans le contexte difficile de la réforme licencemaster-doctorat (LMD), mise en en place dans le cadre de l'harmonisation européenne des cursus universitaires en cours de développement, il est indispensable que les avancées significatives effectuées en matière d'enseignement de l'anglais aux futurs professionnels de la santé ne soient pas remises en cause car cet enseignement a fait la preuve de sa qualité et de son efficacité.

\section{Contributions}

Félicie Pastore a rédigé l'article ; elle est responsable du projet «Synergie ANEMF/GERAS » au sein du groupe «santé » du Groupe d'étude et de recherche en anglais de spécialité (GERAS). Rita Camaré a encouragé et soutenu une réforme profonde de l'enseignement de l'anglais à la faculté de médecine Toulouse-Purpan. Jonathan Broner a manifesté un soutien constant auprès des étudiants, qui a constitué un élément essentiel dans la réussite du projet. Maelle Allibe a contribué à la rédaction de la charte d'enseignement de l'anglais et a contribué de façon déterminante à sa promotion au niveau national.

\section{Références}

1. Pastore F. Enseigner l'anglais à des professionnels de la santé dans le contexte de l'émergence de la pédagogie médicale. Mémoire pour l'obtention du Diplôme d'études approfondies, Université de Bordeaux 2, 1999.

2. Converset-Dumont Y. L'environnement matériel et humain comme paramètre constitutif des choix pédagogiques des enseignants : étude de cas en facultés de médecine. Mémoire pour l'obtention du Diplôme d'études approfondies, Université de Bordeaux 2, 2002.

3. Pastore F. English in French-speaking medical schools: a survey. In: Felix Mayer (Ed.). Language for special purposes: perspectives for the new millennium (Vol. 2) Tübingen: Gunter Narr Verlag; 2001: 853-4.

4. Pastore F. Résultats de l'enquête : problématique de l'enseignement de l'anglais dans les facultés de médecine francophones; triple prise de point de vue. $\mathrm{XIII}^{\mathrm{e}}$ Journées Universitaires Francophones de Pédagogie Médicale Université de Nantes, France, 1999. In : Rev Educ Méd 1999; 13 (1,nº spécial) 1999. 199 [On-line] Disponible sur : http://www .cidmef. u-bordeaux2.fr/WNantes/vol_com.pdf.

5. Rannou F, Charpy J-P, Carnet D, Poiraudeau S, Hamonet M-A. Pratique de l'enseignement de l'anglais dans les facultés de médecine en France métropolitaine. Presse Med 2007;36:794-8.

6. Groupe d'études et de recherche en anglais de spécialité (GERAS). Compte-rendu de la présentation du projet d'harmonisation interministériel de l'enseignement de l'anglais médical au bureau de la Conférence des doyens 
des facultés de médecine, 19 octobre 2004 [On-line] Disponible sur : http://www.geras.fr/dossiers/ dossiers.php?val=93_reunion+intermediaire.

7. Carnet D, Morgan S, Pastore F. Une idée novatrice en formation médicale continue : le DIVAM. Diplôme Inter universitaire d'Anglais pour la Médecine. Besoin superflu ou nécessité impérieuse ? Presse Med 1999;28:14747.

8. Carnet D, Morgan S, Pastore F. A new treatment developed by a team of specialists from the language laboratories of Dijon, Nice and Nantes ASp Anglais de Spécialité 1999(n 23/26):603-7.

9. Journées d'été de l'ANEMF. Améliorer l'enseignement de l'anglais au cours du cursus médical. 2006 [Online] Disponible sur : http://www.geras.fr/ bibliotheque/File/2007ANEMF . doc.

10. Neufeld VR, Woodward CA, MacLeod SM. The McMaster M.D. program: a case study of renewal in medical education. Acad Med 1989;64:423-32.

11. Guilbert J-J. Comment raisonnent les médecins. Réflexions sur la formation médicale. Genève : Éditions Médecine et Hygiène, 1992.

12. Ferland J-J. Les grandes questions de la pédagogiemédicale : perspective nord-américaine. Laval(QC) : Les presses de l'Université, 1987.
13. Kaufmann D. Le nouveau paradigme dans l'enseignement médical : comment la théorie peut exercer une influence sur la pratique. XIII ${ }^{\mathrm{e}}$ Journées Universitaires Francophones de Pédagogie Médicale Université de Nantes (France), 1999. In : Rev Educ Méd 1999 ; 13 (1, $\mathrm{n}^{\circ}$ spécial). 1999 [On-line] Disponible sur : http:// www. cidmef.u-bordeaux2. fr/WNantes/vol_com . pdf.

14. Carnet D, Morgan S, Pastore F. L'anglais pour la santé. Exercices d'appropriation de la langue à visée professionnelle. Paris : Ellipses, 2001 (nouvelle édition 2008).

15. Diplôme Inter Universitaire d'Anglais pour la Santé. 2009 [On-line] Disponible sur : http://www.medecine. ups-tlse. fr/anglais/index.php?pg=8.

16. Carnet D, Foucher G, Jeannin L. L'Anglais médical à la Faculté : l'expérience dijonnaise. Presse Med 1997;26:1200-2.

17. Association Nationale des Étudiants en Médecine de France (ANEMF) et Groupe d'Étude et de Recherche en Anglais de Spécialité (GERAS). Charte « Enseignement de l'anglais dans les facultés de médecine en France métropolitaine » 2007 [On-line] Disponible sur : http: // www medecine.ups-tlse. fr/anglais/index . php?pg=1.

Correspondance et offprints : Félicie Pastore, Faculté de Médecine, 37 allées Jules Guesde, BP 7202, 31073 Toulouse Cedex 7, France.

Mailto : pastore@cict.fr 